\title{
Classical and Quantum Solutions in Brans-Dicke Cosmology with a Perfect Fluid
}

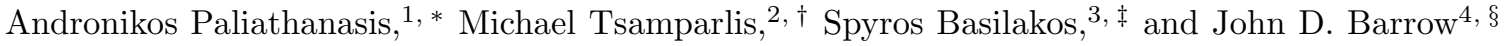 \\ ${ }^{1}$ Instituto de Ciencias Físicas y Matemáticas, Universidad Austral de Chile, Valdivia, Chile \\ ${ }^{2}$ Faculty of Physics, Department of Astrophysics - Astronomy - Mechanics, \\ University of Athens, Panepistemiopolis, Athens 157 83, Greece \\ ${ }^{3}$ Academy of Athens, Research Center for Astronomy and Applied Mathematics, Soranou Efesiou 4, 11527, Athens, Greece \\ ${ }^{4}$ DAMTP, Centre for Mathematical Sciences, University of Cambridge, Wilberforce Rd., Cambridge CB3 OWA, UK
}

\begin{abstract}
We consider the application of group invariant transformations in order to constrain a flat isotropic and homogeneous cosmological model, containing of a Brans-Dicke scalar field and a perfect fluid with a constant equation of state parameter $w$, where the latter is not interacting with the scalar field in the gravitational action integral. The requirement that the Wheeler-DeWitt equation be invariant under one-parameter point transformations provides us with two families of power-law potentials for the Brans-Dicke field, in which the powers are functions of the Brans-Dicke parameter $\omega_{B D}$ and the parameter $w$. The existence of the Lie symmetry in the Wheeler-DeWitt equation is equivalent to the existence of a conserved quantity in field equations and with oscillatory terms in the wavefunction of the universe. This enables us to solve the field equations. For a specific value of the conserved quantity, we find a closed-form solution for the Hubble factor, which is equivalent to a cosmological model in general relativity containing two perfect fluids. This provides us with different models for specific values of the parameters $\omega_{B D}$, and $w$. Finally, the results hold for the specific case where the Brans-Dicke parameter $\omega_{B D}$ is zero, that is, for the O'Hanlon massive dilaton theory, and consequently for $f(R)$ gravity in the metric formalism.
\end{abstract}

PACS numbers: 98.80.-k, 95.35.+d, 95.36.+x

Keywords: Cosmology; Brans-Dicke; Wheeler-DeWitt; Group invariant transformations.

\section{INTRODUCTION}

The comprehensive analysis of various observational data (Cosmic Microwave Background, Supernova Type Ia, large-scale structures, etc.) supports a picture in which the universe is spatially flat with only about $30 \%$ of its total energy in the form of dark or luminous forms of matter. The nature of the remaining $70 \%$, residing in some unknown form of enigmatic 'dark energy' remains a mystery even though it can be accurately described by a particular type of anti-gravitating stress. Discovering the physics of this dark energy, driving the recent accelerated expansion of the universe, is a key goal of theoretical physics and cosmology. The intense debate among the cosmologists and theoretical physics has opened up the possibility of various new cosmological scenarios which offer different sources for the observed acceleration and even a possibility to link it to the suspected era of inflationary acceleration in the very early universe. Some of these scenarios are based on the existence of new fields in nature, while others modify the classical Einstein-Hilbert action for gravity [1-16].

In Friedmann-Lemaître-Robertson-Walker (FLRW) cosmologies containing scalar fields, some analytical solutions without matter can be found in [17 23]. If a matter component is included in the dynamics then new solutions were also found in 24 27]. Furthermore, a new class of solutions has been found from the application of group invariants, namely Lie/Noether symmetries of the field equations [28, 29]. In fact the idea to use Noether point symmetries in cosmology is not new and indeed there is a lot of work in the literature (see [30]). In some previous papers we have provided the Lie/Noether symmetries for various cosmological models, including scalar fields [28], $f(R)$ [31], $f(T)$ 32] and scalar tensor theories 33]. Recently, we have used this dynamical symmetry approach in order to provide solutions to the WdW equation [34].

In general for scalar-tensor theories, including the particular case of Brans-Dicke gravity [10], various analytical solutions are available in the literature [35 37]. In Ref.[46], an exact solution describes a Brans-Dicke scalar field which interacts with a perfect fluid in the action integral. Moreover, some closed-form solutions for plane symmetric spacetimes can be found in [38], and some black-hole solutions in Brans-Dicke gravity are given in [39 [41]. Using the

\footnotetext{
*Electronic address: anpaliat@phys.uoa.gr

$\dagger$ Electronic address: mtsampa@phys.uoa.gr

${ }^{\ddagger}$ Electronic address: svasil@academyofathens.gr

$\S$ Electronic address: jdb34@hermes.cam.ac.uk
} 
method of group invariants, some new exact solutions without matter source are found in [43 45], and with dust in [33].

The purpose of this paper is to extend the method proposed in Ref. 34 to Brans-Dicke gravity when the perfect fluid does not interact with the Brans-Dicke field in the action integral. The proposed selection rule determines the potential that defines the Brans-Dicke field in order for the Wheeler-DeWitt (WdW) equation to be invariant under a group of point transformations. In [34] that proposed method was applied in general relativistic cosmology for a homogeneous scalar field and a perfect fluid. It has been shown that when the $\mathrm{WdW}$ equation is invariant under one-parameter point transformation then, the WdW equation can be solved by separation of variables. The solution provides oscillatory terms in the wavefunction and, at the same time, the point transformations give Noetherian conservation laws for the classical field equations. This latter property can be used to study the integrability of the field equations and extract closed-form solutions.

The structure of the paper is as follows. In Section 2 we present the field equations in Brans-Dicke gravity. In Section 3 we apply Lie point symmetries to the WdW equation and, in section 4 , we provide the invariant solution of the WdW equation. Also, in the same section, we use the Hamilton-Jacobi theory to reduce the field equations to a pair of first-order differential equations and under specific conditions (the Noetherian conservation law vanishes) we obtain a closed-form solution for the Hubble parameter in Brans-Dicke gravity. Then we check the performance of this special Brans-Dicke model against the latest observational data. Finally, in Section 5 we discuss our results and we draw our conclusions.

\section{FIELD EQUATIONS IN BRANS-DICKE GRAVITY}

In Brans-Dicke gravity the gravitational action in the Jordan frame and matter that is not interacting with the Brans-Dicke field is defined by [15]

$$
S=\int d x^{4} \sqrt{-g}\left[\frac{1}{2} \phi R-\frac{1}{2} \frac{\omega_{B D}}{\phi} g^{\mu \nu} \phi_{; \mu} \phi_{; \nu}-V(\phi)\right]+\int d x^{4} \sqrt{-g} L_{m},
$$

where $L_{m}$ is the Lagrangian of the matter, $\phi$ is the Brans-Dicke scalar field, and $\omega_{B D}$ is the Brans-Dicke parameter.

Variations of $S$ (11) with respect to the metric tensor and the field $\phi$ gives the modified Einstein field equations

$$
\phi G_{\mu \nu}=\frac{\omega_{B D}}{\phi}\left(\phi_{; \mu} \phi_{; \nu}-\frac{1}{2} g_{\mu \nu} g^{\kappa \lambda} \phi_{; \kappa} \phi_{; \lambda}\right)-g_{\mu \nu} V(\phi)-\left(g_{\mu \nu} g^{\kappa \lambda} \phi_{; \kappa \lambda}-\phi_{; \mu} \phi_{; \nu}\right)+k T_{\mu \nu},
$$

and the Klein-Gordon equation

$$
g^{\mu \nu} \phi_{; \mu \nu}-\frac{1}{2 \phi} g^{\mu \nu} \phi_{; \mu} \phi_{; \nu}+\frac{\phi}{2 \omega_{B D}}\left(R-2 V_{, \phi}\right)=0,
$$

where $k \equiv 8 \pi G$. In (2) $T_{\mu \nu}$ is the energy-momentum tensor of matter. Since we have assumed that the matter is not interacting with the Brans-Dicke field, we have the conservation law $T_{; \nu}^{\mu \nu}=0$.

In the following we study the solution of these equations under the following assumptions.

a. Spacetime is spatially flat with FLRW metric line element

$$
d s^{2}=-d t^{2}+a^{2}(t)\left(d x^{2}+d y^{2}+d z^{2}\right)
$$

whose Ricci scalar is

$$
R=6\left[\frac{\ddot{a}}{a}+\left(\frac{\dot{a}}{a}\right)^{2}\right] .
$$

b. Matter is a perfect fluid for comoving observers $u_{\mu}=\delta_{\mu}^{0}$; that is, the energy-momentum tensor is

$$
T_{\mu \nu}=\left(\rho_{m}+p_{m}\right) u_{\mu} u_{\nu}+p_{m} g_{\mu \nu}
$$

where $\rho_{m}$, is the energy density of the matter and $p_{m}$ is the isotropic pressure measured by the observers $u_{\mu}$.

c. The perfect fluid has a constant equation of state (EoS) parameter $w$, i.e., $p_{m}=w \rho_{m}$. For a barotropic fluid $w \in[0,1]$; for $w=0, T_{\mu \nu}$, describes dust and for $w=1 / 3$, a radiation fluid. In what follows we will extend the range 
of the EoS parameter to be $w \in(-1,1)$ and consider effects 'fluids'. Of course, the lower limit, $w=-1$, corresponds to a cosmological constant which can always be absorbed in the scalar-field potential.

d. We assume that the scalar field, $\phi$, possesses the same symmetries as the spacetime, that is $\phi(t, x, y, z) \equiv \phi(t)$.

From the conservation law $T_{; \nu}^{\mu \nu}=0$ we find that $\rho_{m}=\rho_{m 0} a^{-3(1+w)}$, where $\rho_{m 0}$ is the energy density of today, and $\rho_{m 0}=3 \Omega_{m 0} H_{0}^{2}$. Under these assumptions the Lagrangian of the field equations becomes

$$
\mathcal{L}(a, \dot{a}, \phi, \dot{\phi})=-3 a \phi \dot{a}^{2}-3 a^{2} \dot{a} \dot{\phi}+\frac{1}{2} \frac{\omega_{B D}}{\phi} a^{3} \dot{\phi}^{2}-a^{3} V(\phi)-k \rho_{m 0} a^{-3 w}
$$

and the first Brans-Dicke Friedmann equation is

$$
3 H^{2}=\frac{\omega_{B D}}{2}\left(\frac{\dot{\phi}}{\phi}\right)^{2}+\frac{V(\phi)}{\phi}-3 H \frac{\dot{\phi}}{\phi}+\frac{k}{\phi} \rho_{m 0} a^{-3(1+w)},
$$

where $H=\dot{a} / a$, is the Hubble function, and an overdot indicates differentiation with respect to the comoving proper time coordinate $t$.

In general, the Lagrangian (7) defines the motion of a particle in a two-dimensional space $\{a, \phi\}$ with effective potential

$$
V_{e f f}=a^{3} V(\phi)+k \rho_{m 0} a^{-3 w}
$$

For $\omega_{B D} \neq-3 / 2$, from Lagrangian ${ }^{1}(7)$ we define the momentum, $p_{a}=\frac{\partial L}{\partial \dot{a}}, p_{\phi}=\frac{\partial L}{\partial \dot{\phi}}$, hence we can write the Hamiltonian

$$
\mathcal{E}=\frac{1}{2 \omega_{B D}+3}\left[-\frac{\omega_{B D}}{2 a \phi} p_{a}^{2}-\frac{3}{a^{2}} p_{a} p_{\phi}+\frac{3 \phi}{a^{3}} p_{\phi}^{2}\right]+a^{3} V(\phi)+k \rho_{m 0} a^{-3 w}
$$

From the first modified Friedmann equation (8) it follows that $\mathcal{E}=0$.

Therefore, the second modified Friedmann equation and the Klein Gordon equation are described by the following Hamiltonian system:

$$
\begin{gathered}
\left(2 \omega_{B D}+3\right) \dot{a}=-\frac{2 \omega_{B D}}{3 a \phi} p_{a}-\frac{2}{a^{2}} p_{\phi} \\
\left(2 \omega_{B D}+3\right) \dot{\phi}=-\frac{2}{a^{2}} p_{a}+\frac{4 \phi}{a^{3}} p_{\phi}, \\
\left(2 \omega_{B D}+3\right) \dot{p}_{\phi}=-\frac{\omega_{B D}}{3 a \phi^{2}} p_{a}^{2}-\frac{2}{a^{3}} p_{\phi}^{2}-\left(2 \omega_{B D}+3\right) a^{3} V_{,}, \\
\left(2 \omega_{B D}+3\right) \dot{p}_{a}=-\frac{\omega_{B D}}{3} \frac{p_{a}^{2}}{a^{2} \phi}-\frac{4}{a^{3}} p_{a} p_{\phi}+\frac{6 \phi}{a^{4}} p_{\phi}^{2}+ \\
-3\left(2 \omega_{B D}+3\right)\left(a^{2} V(\phi)-k \rho_{m 0} w a^{-1-3 w}\right) .
\end{gathered}
$$

Under normal quantization, i.e., $p_{i} \simeq i \frac{\partial}{\partial x^{i}}$, we can define the $\mathrm{WdW}$ equation $W:=\mathcal{E}(\Psi)=0$, that is 48 , 49],

$$
\begin{aligned}
0 & =\frac{1}{6\left(2 \omega_{B D}+3\right)}\left[\frac{\omega}{a \phi} \Psi_{, a a}-\frac{6}{a^{2}} \Psi_{, a \phi}+\frac{6 \phi}{a^{3}} \Psi_{, \phi \phi}\right]+ \\
& +\frac{1}{6\left(2 \omega_{B D}+3\right)}\left[\frac{6}{a^{3}} \Psi_{, \phi}-\frac{\omega}{a^{2} \phi} \Psi_{, a}\right]-\left[a^{3} V(\phi)+k \rho_{m 0} a^{-3 w}\right] \Psi,
\end{aligned}
$$

\footnotetext{
${ }^{1}$ In the limit in which $\omega_{B D}=-3 / 2$, lagrangian (7) is denerate, i.e. $\left|\frac{\partial^{2} L}{\partial \dot{x}^{i} \partial \dot{x}^{j}}\right|=0$, for a discussion see [47].
} 
where $\Psi=\Psi(a, \phi)$ indicates the wavefunction of the universe. Notice, that we use the following derivatives $\Psi, a a=$ $\partial^{2} \Psi / \partial a^{2}, \Psi_{\phi \phi}=\partial^{2} \Psi / \partial \phi^{2}$ and $\Psi_{, a \phi}=\partial^{2} \Psi / \partial a \partial \phi$. Recall that the dimension of the minisuperspace is two: that is, we do not introduce the quantum correction term in order for the WdW equation to be conformally invariant [50, 51].

The WdW equation is defined by the conformally invariant operator

$$
\hat{L}_{\gamma}=-\Delta_{\gamma}+\frac{n-2}{4(n-1)} R_{\gamma}
$$

where $\Delta_{\gamma}$ is the Laplace operator with respect to the minisuperspace $\gamma_{i j}$, and $R_{\gamma}$, is the Ricci scalar of $\gamma_{i j}$. The importance of the operator $\hat{L}_{\gamma}$ is that under a conformal transformation, $\bar{\gamma}_{i j}=e^{2 \Omega\left(x^{k}\right)} \gamma_{i j}$, the scaling $\hat{L}_{\bar{\gamma}}(\Psi)=$ $e^{-\frac{n+2}{2} \Omega\left(x^{k}\right)} \hat{L}_{\gamma}\left(e^{\frac{n-2}{2} \Omega\left(x^{k}\right)} \Psi\right)$ holds, where $\Omega\left(x^{k}\right)$ is an arbitrary function. Moreover, using the the kinetic term in the Lagrangian (7), we endow the minisuperspace $\gamma_{i j}$ (the dimension is $n=2$ ), with line element:

$$
d s_{(\gamma)}^{2}=-6 a \phi d a^{2}-6 a^{2} d a d \phi+\frac{\omega_{B D}}{\phi} a^{3} d \phi^{2}
$$

from which we conform that the associated Ricci scalar vanishes, i.e. $R_{\gamma}=0$.

Hence, from (16), it follows that $L_{\gamma}=-\Delta_{\gamma}$, which is the Laplace operator, and under a conformal transformation we have $L_{\bar{\gamma}}(\Psi)=e^{-2 \Omega\left(x^{k}\right)} L_{\gamma}(\Psi)$. In order to solve (15), we need to specify the scalar-field potential. This will be done by an ansatz. In the literature there have been many forms for this potential depending on what one wants to do. In the present work we adopt the geometric approach to dictate the physics. The gain from this approach is that it is observer-free and no conflicts arise between geometry and dynamics. Specifically, we require that the potential $V(\phi)$ be such that the WdW equation (15) admits Lie point symmetries.

\section{GROUP-INVARIANT TRANSFORMATIONS FOR THE WDW EQUATION}

For convenience, we provide below the basic definitions of Lie point symmetries. Let $W=W\left(x^{i}, \Psi, \Psi_{, i}, \Psi_{, i j}\right)$ be a second-order differential equation, $x^{i}$ are the independent variables, and $\Psi$ is the dependent variable, where $\Psi_{, i}=\frac{\partial \Psi}{\partial x^{i}}$ and $\Psi_{, i j}=\frac{\partial^{2} \Psi}{\partial x^{i} \partial x^{j}}$. The generator $X$ of the infinitesimal one-parameter point transformation

$$
\bar{x}^{i}=x^{i}+\varepsilon \xi^{i}\left(x^{i}, \Psi\right), \bar{\Psi}=\Psi+\varepsilon \eta\left(x^{i}, \Psi\right)+O\left(\varepsilon^{2}\right)
$$

is defined by

$$
X=\frac{\partial \bar{x}^{i}}{\partial \varepsilon} \partial_{i}+\frac{\partial \bar{\Psi}}{\partial \varepsilon} \partial_{\Psi},
$$

from which it follows that

$$
X=\xi^{i}\left(x^{i}, \Psi\right) \partial_{i}+\eta\left(x^{i}, \Psi\right) \partial_{\eta}
$$

The differential equation, $W$, is invariant under the action of the one-parameter point transformation (18) if there exists a function $\kappa$ such that 52,53 .

$$
X^{[2]} W=\kappa W
$$

where $X^{[2]}$ is the second prolongation of $X$ in the jet space $\left\{x^{i}, \Psi, \Psi_{, i}, \Psi_{, i j}\right\}$. When condition (21) holds, we say that $X$ is a Lie point symmetry of $W$. Notice, that the Lie point symmetries of a differential equation form a Lie algebra.

For differential equations which follow from a variational principle, i.e. there exists a Lagrange function, the Lie point symmetries which transform the action integral in such a way that the Euler-Lagrange equations are invariant are called Noether point symmetries. The characteristic of Noether point symmetries is that for each Noether symmetry, $X$, there corresponds a conservation law which is called a Noether integral [54]. The Noether point symmetries of a differential equation form a subalgebra of the Lie point symmetries of that equation which is called the Noether algebra of the differential equation. 


\subsection{Lie point symmetries of the $\mathrm{WdW}$ equation}

The WdW equation (15) is a second-order partial differential equation defined in the space of the independent variables $\left\{x^{i}\right\} \rightarrow\{a, \phi\}$, where $\Psi$ is the dependent variable. Hence, the generator (20) of the infinitesimal point transformation (18) has the following form,

$$
X=\xi^{a}(a, \phi, \Psi) \partial_{\alpha}+\xi^{\phi}(a, \phi, \Psi) \partial_{\phi}+\eta(a, \phi, \Psi) \partial_{\Psi} .
$$

From condition (21), and for arbitrary $V(\phi)$, we have the following Lie symmetries:

$$
X_{\Psi}=\Psi \partial_{\Psi}, X_{b}=b(a, \phi) \partial_{\Psi}
$$

where $b(a, \phi)$ is a solution of the original equation. The vector field $X_{\Psi}$ is called a homogeneous symmetry, whereas $X_{b}$ corresponds to the infinite number of solutions. Both these Lie symmetries are trivial symmetries in the sense that they cannot be used to reduce the differential equation. However, they indicate that equation (15) is a linear second-order partial differential equation.

In order that equation (15) admit non trivial Lie symmetries, we must consider specific forms of the potential $V(\phi)$. In [34, 55] it has been shown that the WdW equation (15) admits nontrivial Lie point symmetries if and only if the potential $V(\phi)$ is power law, with

$$
V(\phi)=V_{0} \phi^{\lambda}
$$

In our case the power $\lambda=\lambda\left(\omega_{B D}, w\right)$ has the following possible values: (a) $\lambda_{1}=(1+w)(1-w)^{-1}$, and, (b) $2 \lambda_{2}=(\varpi-3)(w+1)$, where

$$
\varpi=\sqrt{6 \omega_{B D}+9} .
$$

The Lie point symmetry vector which corresponds to $\lambda_{1}$ is ${ }^{2}$

$$
X_{1}=a \partial_{a}+3(w-1) \phi \partial_{a}
$$

and to $\lambda_{2}$ is,

$$
X_{2}=A^{\mu_{1}} \phi^{\mu_{2}}\left(a \partial_{a}+\frac{6 \phi}{\varpi-3} \partial_{\phi}\right),
$$

where $\mu_{1}=\frac{3}{2 \varpi}[\varpi(w-1)-3 w+1]$ and $\mu_{2}=\frac{\varpi+3}{2} \mu_{1}$.

We remark that the vector fields $X_{1}, X_{2}$, are conformal symmetries for the minisuperspace $\gamma$ defined by the kinematic part of the Lagrangian (77). Therefore, the minisuperspace selects the form of the potential [28, 34, 55]. A special case occurs when the perfect fluid is radiation, that is, $w=1 / 3$. In this case we find another power-law potential of the form

$$
V_{w=1 / 3}(\phi)=V_{0}\left[\left(V_{1}-\phi^{-\frac{1}{3} \varpi}\right)^{2}+V_{1}\left(2-\phi^{\frac{1}{3} \varpi}\right)^{2}-V_{1}\right] .
$$

For this potential the WdW equation admits the Lie point symmetry which is given by the vector field, $Y=Y_{+}+$ $Y_{-}$, where

$$
Y_{ \pm}=\phi^{-\frac{1}{2}\left(1 \pm \frac{\varpi}{3}\right)}\left(\partial_{a}+\frac{\varpi \pm 3}{\omega_{B D}} \frac{\phi}{a} \partial_{\phi}\right) .
$$

The importance of the existence of a nontrivial Lie point symmetry for equation (15) is the existence of a coordinate system in which equation (15) is independent of one of the independent variables, and the solution which corresponds to the zero-order invariants has oscillatory terms. Furthermore, a Noetherian conservation law for the field equations (10)-(14) means that they define an integrable Hamiltonian system [34]. In the next section we use the zero-order invariance on the $\mathrm{WdW}$ equation (15) to reduce the field equations to two first-order ordinary differential equations, which we solve.

\footnotetext{
${ }^{2}$ Recall that we have considered $w \in(-1,1)$. In the limit where $w=1$, only the power law potential with $\lambda_{2}$ exists.
} 


\section{ANALYTICAL SOLUTIONS}

Now we apply the zero-order invariants of the Lie point symmetries for the WdW equation in order to reduce the equation and find the solution of the wavefunction $\Psi(a, \phi)$. Moreover, by using the Hamilton-Jacobi equation we reduce the dimension of the Hamiltonian system for the field equations. We do that for the potential (24) with $\lambda=\lambda_{1}$.

\subsection{Invariant solution for the WdW equation}

In order to apply the zero-order invariants of a Lie point symmetry in equation (15), we prefer to work with the normal coordinates of the symmetry vector $X_{1}$. We apply the coordinate transformations

$$
a=\exp (x), \phi=y \exp [3(w-1) x]
$$

to (15) and the WdW equation takes the following form

$$
\begin{aligned}
0= & \left(-\frac{\omega_{B D}}{3 y} \Psi_{, x x}+2 m_{1} \Psi_{, x y}-y m_{2} \Psi_{, y y}\right)+ \\
& -m_{2} \Psi_{, y}-2\left(\bar{V}_{0} y^{\frac{1+w}{1-w}}+\bar{\rho}_{m 0}\right) \Psi
\end{aligned}
$$

where now we have $\Psi=\Psi(x, y), m_{1}=3 \omega_{B D}(w-1)-1, m_{2}=3 \omega_{B D}(w-1)^{2}-2(3 w-2)$ and $\left(\bar{V}_{0}, \bar{\rho}_{m 0}\right)=$ $\left(2 \omega_{B D}+3\right)\left(V_{0}, k \rho_{m 0}\right)$.

In the new coordinates the Lie point symmetry vector $X_{1}$, takes the simple form $X_{1}=\partial_{x}$. Since $X_{1}$ is a Lie symmetry of (31) this means that it transforms solutions to solutions; that is,

$$
X_{1}(\Psi)=\beta \Psi,
$$

from where it follows that, ${ }^{3}$

$$
\Psi(x, y)=\sum_{\beta} e^{-\beta x} \Phi(y)
$$

where the function, $\Phi(y)$, is given by the following second-order ordinary differential equation:

$$
y m_{2} \Phi_{, y y}+\left(2 \beta m_{1}+m_{2}\right) \Phi_{, y}+2\left(\bar{V}_{0} y^{\frac{1+w}{1-w}}+\bar{\rho}_{m 0}+\frac{\omega_{B D}}{3 y} \beta^{2}\right) \Phi=0 .
$$

In fig. 1 we present the wavefunction (33), in the case of the O'Hanlon theory, i.e. with $\omega_{B D}=0$; which can be seen as an effective $f(R)$ gravity with $\phi=\frac{d f}{d R}$ and $V(\phi)=\left(\frac{d f}{d R} R-f\right)$ [57]. Specifically, in the left panels of fig. [1 we show the solutions of $\operatorname{Re}(\Psi(a, \phi))$ and $\operatorname{Im}(\Psi(a, \phi))$ respectively, for $\rho_{m 0}=0$, and $w=1 / 3$. The latter case is for the the radiation-dominated era. As we have discussed above, the previously selected dynamical conditions imply $V(\phi)=V_{0} \phi^{2}$, hence we can easily show that the corresponding ${ }^{4} f(R)$-theory is $f(R) \propto R^{2}$. We would like to remind the reader that the $R^{2}$ term provides a de-Sitter behavior [58] which plays a critical role in the inflationary era. In fact, if the gravitational Lagrangian is $f(R)$, there exist de Sitter solutions of the theory with covariantly constant $R_{0}$ if it is a solution of Barrow and Ottewill's condition $R_{0} f^{\prime}\left(R_{0}\right)=2 f\left(R_{0}\right)$, [59], which is satisfied identically for the purely quadratic Lagrangian. Notice, that in the right panels of fig. 1, we plot the contours of the wavefunction in the $(a, \phi)$ plane.

Now we focus on Eq.(34). In the special case of $m_{2}=0$, namely $\omega_{B D}=\frac{2}{3} \frac{(3 w-2)}{(1-w)^{2}}$, the corresponding solution is

$$
\Phi(y)=\Phi_{0} \exp \left[-\bar{V}_{0} \frac{(1-w)}{2 \beta m_{1}} y^{\frac{2}{1-w}}-\frac{\bar{\rho}_{m 0}}{\beta m_{1}} y-\frac{\beta}{6 m_{1}} \ln y\right]
$$

\footnotetext{
${ }^{3}$ We take the same result when we apply the zero-order invariants of the symmetry vector $Z=X_{1}-\beta X_{\Psi}$ in (31).

${ }^{4}$ In $f(R)$-theory, in the metric formalism the gravitational action integral is $S=\int d x^{4} \sqrt{-g} f(R)$, where $R$ is the Ricci scalar of the underlying space with metric $g_{i j}$.
} 

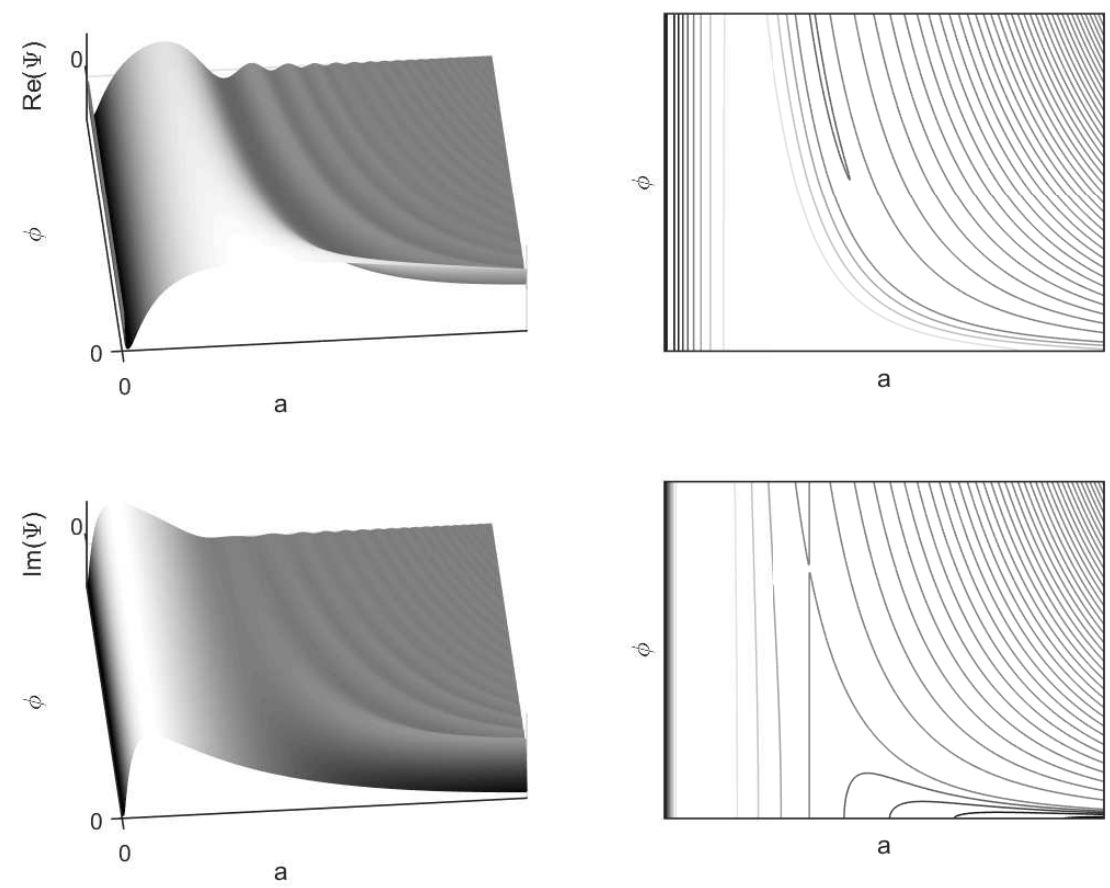

FIG. 1: Left panels: The surface plot of the wavefunction (33) for $\omega_{B D}=0, \bar{\rho}_{m 0}=0, w=1 / 3$ and $\beta=1 i$, which corresponds to the quadratic potential $V(\phi)=V_{0} \phi^{2}$. Notice that we use $V_{0}=1$ units. The current dynamical model effectively reduces to $f(R) \propto R^{2}$ gravity. Right panels: The contours of the wavefunction in the $(a, \phi)$ plane.

In the next section we continue with the classical solution of the field equations. As we shall see, the constant $\beta$ is related to the value of the Noetherian conservation law for the field equations, which corresponds to the vector field $X_{1}$.

\subsection{Classical solution}

In the coordinate system (30) the Hamiltonian of the field equations (10) becomes

$$
\mathcal{E}=\frac{e^{-3 w x}}{6}\left(-\frac{\omega_{B D}}{y} p_{x}^{2}+6 m_{1} p_{x} p_{y}-3 y m_{2} p_{y}^{2}\right)+e^{-3 w x}\left(\bar{V}_{0} y^{\frac{1+w}{1-w}}+\bar{\rho}_{m 0}\right)
$$

and the field equations are given by the following Hamiltonian system

$$
\begin{gathered}
e^{3 w x} \dot{x}=m_{1} p_{y}-\frac{\omega_{B D}}{3 y} p_{x}, \\
e^{3 w x} \dot{y}=m_{1} p_{x}-m_{2} y p_{y}, \\
e^{3 w x} \dot{p}_{x}=3 w \mathcal{E}, \\
e^{3 w x} y^{2} \dot{p}_{y}=\frac{1}{2} m_{2} y^{2} p_{y}^{2}+\frac{1}{6} \omega_{B D} p_{x}^{2}-V_{0} \frac{(1+w)}{(1-w)} y^{\frac{2}{1-w}} .
\end{gathered}
$$

From the first modified Friedmann equation, and equation (39), we have that

$$
p_{x}=I_{0} .
$$


This is the Noetherian conservation law which corresponds to the symmetry vector ${ }^{5} X_{1}$. Comparing the last expression with (32) we see that $|\beta| \simeq\left|I_{0}\right|$.

From (36) we define the (null) Hamilton-Jacobi equation: ${ }^{6}$

$$
\frac{1}{6}\left(-\frac{\omega_{B D}}{y}\left(\frac{\partial S}{\partial x}\right)^{2}+6 m_{1}\left(\frac{\partial S}{\partial x}\right)\left(\frac{\partial S}{\partial y}\right)-3 y m_{2}\left(\frac{\partial S}{\partial y}\right)^{2}\right)+\left(\bar{V}_{0} y^{\frac{1+w}{1-w}}+\bar{\rho}_{m 0}\right)=0
$$

where $S=S(x, y)$ is the action. Furthermore, from (41), we have the constraint $\left(\frac{\partial S}{\partial x}\right)=I_{0}$. Hence, from this and from (42) we find,

$$
\begin{aligned}
S(x, y) & =I_{0} x+\frac{m_{1}}{m_{2}} I_{0} \ln (y)+ \\
& +\int \sqrt{I_{0}^{2} y^{-2}\left[\left(\frac{m_{1}}{m_{2}}\right)^{2}-\frac{\omega_{B D} \omega}{m_{2}}\right]+2 m_{2}^{-1}\left(\bar{V}_{0} y^{\frac{2 w}{1-w}}+\bar{\rho}_{m 0} y^{-1}\right) d y}
\end{aligned}
$$

while for $m_{2}=0$, the action $S(x, y)$ becomes

$$
S(x, y)=I_{0} x+\frac{\omega_{B D} I_{0}}{6 m_{1}} \ln (y)-\frac{1}{2}\left(\bar{V}_{0}(1-w) y^{\frac{2}{1-w}}+\frac{\bar{\rho}_{m 0}}{I_{0} m_{1}} y\right) .
$$

Hence, the field equations (37)-(40) reduce to the following two-dimensional system of first-order differential equations:

$$
\begin{gathered}
e^{3 w x} \dot{x}=m_{1}\left(\frac{\partial S}{\partial y}\right)-\frac{\omega_{B D}}{3 y}\left(\frac{\partial S}{\partial x}\right), \\
e^{3 w x} \dot{y}=m_{1}\left(\frac{\partial S}{\partial x}\right)-m_{2} y\left(\frac{\partial S}{\partial y}\right),
\end{gathered}
$$

where, $S=S(x, y)$, is given by (43) or (44). In general, the solution of the system (45)-(46), is not given in closed form. Below, for the specific case where the Noetherian conservation law vanishes, we can express the solution in terms of the scale factor.

\subsection{A special closed-form solution}

We consider now the case where $I_{0}=0$, i.e. $p_{x}=0$ with $m_{1} \neq 0$, From (37) and (38) we have $\frac{d y}{d x}=-\frac{m_{2}}{m_{1}} y$, so $y=\phi_{0} e^{-\frac{m_{2}}{m_{1}} x}$, hence with the use of (30) for the field $\phi$, we have

$$
\phi(a)=\phi_{0} a^{M}, M=3(w-1)-\frac{m_{2}}{m_{1}}
$$

so $\dot{\phi}=\phi_{0} M a^{M} H$. Then, from (자), it follows that

$$
\left[3(1+M)-\frac{\omega_{B D} M}{2}\right] H^{2}=G_{e f f}\left(V_{0}^{\prime} a^{\frac{1+w}{1-w} M}+k \rho_{m 0} a^{-3(1+w)}\right),
$$

where $V_{0}^{\prime}=V_{0} \phi_{0}^{\frac{1+w}{1-w}}$, and $G_{e f f}=\left[\left(3(1+M)-\frac{\omega_{B D} M}{2}\right) \phi\right]^{-1}$ is the effective gravitational constant. We see that $G_{\text {eff }}(a \rightarrow 1)=\left[\left(3(1+M)-\frac{\omega_{B D} M}{2}\right) \phi_{0}\right]^{-1}$. Therefore, we can say that when $I_{0}=0$, the scalar field behaves like an effective fluid with constant EoS parameter. A special solution of the form, $\phi=\phi_{0} a^{\phi_{1}}$, where $\phi_{1}$ is a constant, has

\footnotetext{
${ }^{5}$ As we can see we did not use the formulas of Noether theorems to calculate the conservation law. However we derived it from the Hamilton equations in the canonical coordinates of the vector field $X_{1}$.

${ }^{6}$ Recall that the action $S(x, y)$ is related to the momenta, $p_{x}=\frac{\partial S}{\partial x}$, and $p_{y}=\frac{\partial S}{\partial y}$.
} 
been found in [46], where $V_{0}=0$ and the perfect fluid is interacting with the scalar field in the action integral. A similar result has been found in [34] . In that paper we applied the same geometric selection rule for the scalar field, but in general relativity $\left(G_{e f f}=\right.$ const $)$ as a special solution of the field equations ${ }^{7}$.

By replacing $G_{\text {eff }}(a)$ in (48), we can define the Hubble function as follows

$$
H(a)^{2}=H_{0}^{2}\left(\Omega_{\phi 0} a^{q_{1}}+\Omega_{m 0} a^{q_{2}}\right),
$$

where spatial flatness requires that $\Omega_{\phi 0}+\Omega_{m 0}=1$, since $E(a \rightarrow 1)=1$. Furthermore, the new constants $q_{1}, q_{2}$ are,

$$
\begin{gathered}
q_{1}=\frac{2 M\left(w, \omega_{B D}\right) w}{1-w}, \\
q_{2}=-\left[M\left(w, \omega_{B D}\right)+3(1+w)\right] .
\end{gathered}
$$

Hence, the system (50)-(51) can provide us with a Hubble function for different models of two fluids (49). We study some special cases:

Case (A): Cosmological constant with dust. This means that $\left(q_{1}, q_{2}\right)=(0,-3)$ or $\left(q_{1}, q_{2}\right)=(-3,0)$, from which we have $\left(w, \omega_{B D}\right)=\left(0, \frac{1}{6}\right)$ or $\left(w, \omega_{B D}\right) \simeq(0.28,-0.77)$.

Case (B): Dust with radiation. This requires, $\left(q_{1}, q_{2}\right)=(-3,-4)$ or $\left(q_{1}, q_{2}\right)=(-4,-3)$, hence $\left(w, \omega_{B D}\right) \simeq(0.63,0)$ or $\left(w, \omega_{B D}\right) \simeq(0.55,-1)$.

Case (C): Cosmological constant with radiation fluid This requires $\left(q_{1}, q_{2}\right)=(0,-4)$ or $\left(q_{1}, q_{2}\right)=(-4,0)$, hence $\left(w, \omega_{B D}\right)=(0,0), \quad\left(w, \omega_{B D}\right)=\left(\frac{1}{3}, 0\right)$ or, $\left(w, \omega_{B D}\right)=\left(\frac{1}{3},-\frac{3}{4}\right)$.

Case (D): In the case of $\left(q_{1}, q_{2}\right)=(0,0)$, which implies $\left(w, \omega_{B D}\right)=\left(-1, \frac{1}{6}\right)$ or $\left(w, \omega_{B D}\right)=\left(0,-\frac{4}{3}\right)$, from (49) we have a de Sitter solution.

It is interesting that when we assume a radiation fluid in (49) we have a solution of the system (50)-(51) in which $\omega_{B D}=0$; however, this result is expected since when $\omega_{B D}=0$, the action (1) reduces to O'Hanlon's massive dilaton gravity [56], and consequently to $f(R)$-gravity in the metric formalism, which provides a radiation term [16].

Before we close this section, we should add that for the power-law potential (24) with $\lambda=\lambda_{2}$, one may use the same method to construct the solution of the field equations. We shall not repeat the calculations but we simply say that in this case the canonical coordinate transformation $\{a, \phi\} \rightarrow\{z, r\}$ is given by the following expression:

$$
z=\frac{3-\varpi}{6 \mu_{2}+\mu_{1}(\varpi-3)} a^{-\mu_{1}} \phi^{-\mu_{2}}, r=\phi a^{-\frac{6}{\varpi-3}} .
$$

\subsection{Observational Constraints}

Now we focus on the Hubble parameter (49) in which we have imposed $w=0$. This means that the perfect fluid in the gravitation action (10) is dust. Therefore, from (50) and (51) we have that $q_{2}=-\frac{3 \omega_{B D}+4}{3 \omega_{B D}+1}$ and $q_{1}=0$. Using the above conditions the Hubble parameter becomes

$$
H(a)^{2}=H_{0}^{2}\left[\left(1-\Omega_{m 0}\right)+\Omega_{m 0} a^{-\frac{3 \omega_{B D}+4}{3 \omega_{B D}+1}}\right] .
$$

We mention that from the second term of Eq.(53) one may define an effective equation of state parameter, namely

$$
w_{m}^{(\mathrm{eff})}=\frac{1}{3} \frac{1-6 \omega_{B D}}{\left(3 \omega_{B D}+1\right)} .
$$

Obviously, for $\omega_{B D}=1 / 6$ (or $w_{m}^{(\text {eff })}=0$ ) the above Hubble parameter reduces to that of the concordance $\Lambda$ CDM model.

In order to constrain the Brans-Dicke parameter we perform a joint likelihood analysis using the Type Ia supernova data set of Union 2.1 [60], and the BAO data [61, 62]. Notice, that for the Hubble constant we utilize $H_{0}=$ $69.6 \mathrm{~km} / \mathrm{s} / \mathrm{Mpc}$ 63]. Hence, the overall likelihood function is defined as follows

$$
\mathcal{L}\left(\Omega_{m 0}, \omega_{B D}\right)=\mathcal{L}_{S N I a} \times \mathcal{L}_{B A O}
$$

\footnotetext{
${ }^{7}$ For a different derivation of the same result see [25] 27].
} 
TABLE I: The overall statistical results (using SNIa+BAO) for the $\Lambda$ CDM and Brans-Dicke models respectively. Notice, that in our analyis we use Eq.(53). In the last three colums we present the number of free parameters and the goodness-of-fit statistics.

\begin{tabular}{ccccccccc}
\hline \hline Model & $\Omega_{m 0}$ & $\Omega_{\Lambda}$ & $w_{\Lambda}$ & $w_{m}$ & & $n_{f i t}$ & $\min \left(\chi^{2}\right)$ & $\mathrm{AIC}$ \\
\hline$\Lambda$ CDM & $0.28_{-0.024}^{+0.025}$ & $0.72_{-0.024}^{+0.025}$ & -1 & 0 & & 1 & 564.51 & 566.51 \\
& & & & & & & & \\
& $\Omega_{m 0}$ & $\Omega_{\phi 0}$ & $w_{\phi}$ & $w_{m}^{(\mathrm{eff})}$ & $\omega_{B D}$ & $n_{f i t}$ & $\min \left(\chi^{2}\right)$ & $\mathrm{AIC}$ \\
\hline Brans-Dicke & $0.29_{-0.025}^{+0.032}$ & $0.71_{-0.025}^{+0.032}$ & -1 & $-0.03_{-0.072}^{+0.091}$ & $0.19_{-0.059}^{+0.075}$ & 2 & 564.29 & 568.29 \\
\hline \hline
\end{tabular}

where $\mathcal{L}_{A} \propto e^{-\chi_{A}^{2} / 2}$ which means that the total $\chi^{2}$ is written as

$$
\chi^{2}=\chi_{S N I a}^{2}+\chi_{B A O}^{2}
$$

Lastly, in order to test the performance of the cosmological models against the data we use the Akaike information criterion $\mathrm{AIC}=\min \left(\chi^{2}\right)+2 n_{f i t}$, where $n_{f i t}$ is the number of free parameter [65].

In the case of SNIa the corresponding chi-square parameter is given by ${ }^{8}$

$$
\chi_{S N I a}^{2}=\sum_{i=1}^{N_{S N I a}}\left(\frac{\mu_{o b s}\left(z_{i}\right)-\mu_{t h}\left(z_{i} ; \Omega_{m 0}, \omega_{B D}\right)}{\sigma_{i}}\right)^{2}
$$

where $N_{S N I a}=580, z_{i} \in[0.015,1.414]$ is the observed redshift, $\mu_{o b s}$ is the observed distance modulus and $\mu_{t h}=$ $5 \log D_{L}+25$ with $D_{L}$ denoting the luminosity distance. Furthermore, for BAOs the corresponding chi-square parameter has the following form

$$
\chi_{B A O}^{2}=\sum_{i=1}^{N_{B A O}}\left(\sum_{j=1}^{N_{B A O}}\left[d_{o b s}\left(z_{i}\right)-d_{t h}\left(z_{i} ; \Omega_{m 0}, \omega_{B D}\right)\right] C_{i j}^{-1}\left[d_{o b s}\left(z_{j}\right)-d_{t h}\left(z_{j} ; \Omega_{m 0}, \omega_{B D}\right)\right]\right)
$$

where $N_{B A O}=6$ and $C_{i j}^{-1}$ is the inverse of the covariant matrix in terms of $d_{z}=\frac{l_{B A O}}{D_{V}(z)}[64$. Notice, that the quantity $l_{B A O}\left(z_{\text {drag }}\right)$ is the BAO scale at the drag redshift and $D_{V}(z)$ is the volume distance [62].

In table [1] we present the results of the current statistical analysis while in figure (2) we provide the $1 \sigma, 2 \sigma$, and $3 \sigma$ combined likelihood contours for the Brans-Dicke model [see Eq.(53)]. In particular, we find the following results:

- for the Brans-Dicke model: $\Omega_{m 0}=0.29_{-0.025}^{+0.032}, \omega_{B D}=0.19_{-0.059}^{+0.075}, \min \left(\chi^{2}\right) \simeq 564.29, n_{f i t}=2$ and AIC $\simeq 568.29$.

- for the $\Lambda \mathrm{CDM}$ model: $\Omega_{m 0}=0.28_{-0.024}^{+0.025}, \min \left(\chi_{\Lambda}^{2}\right) \simeq 564.51, n_{f i t}=1$ and $\mathrm{AIC}_{\Lambda} \simeq 566.51$.

Since, $\Delta \mathrm{AIC}=\left|\mathrm{AIC}-\mathrm{AIC}_{\Lambda}\right| \leq 2$ we conclude that the current cosmological models fit equally well the observational data.

\section{CONCLUSIONS}

In this paper, we have extended our earlier analysis, which was introduced in [34], for the case of Brans-Dicke gravity with a perfect fluid in which the fluid EoS parameter is constant and the underlying geometry is that of a spatially flat FLRW universe. In particular, in order to select the functional form of the scalar field potential, $V(\phi)$, in the gravitational action integral (1), we have used the well known criterion, namely the existence of group invariant transformations for the WdW equation [34]. The existence of a Lie point symmetry vector for the WdW equation is related to the existence of oscillatory terms in the solution of the wavefunction $\Psi$, and to Noetherian conservation laws for the field equations. The latter can be used to find analytical solutions.

For our model, in which the perfect fluid is not interacting with the scalar field, we found two families of solutions with power-law potential $V(\phi)=V_{0} \phi^{\lambda}$, where the constant $\lambda$ depends on the EoS parameter, $w$, of the perfect fluid

\footnotetext{
${ }^{8}$ For the SNIa test we have applied the diagonal covariant matrix without the systematic errors.
} 


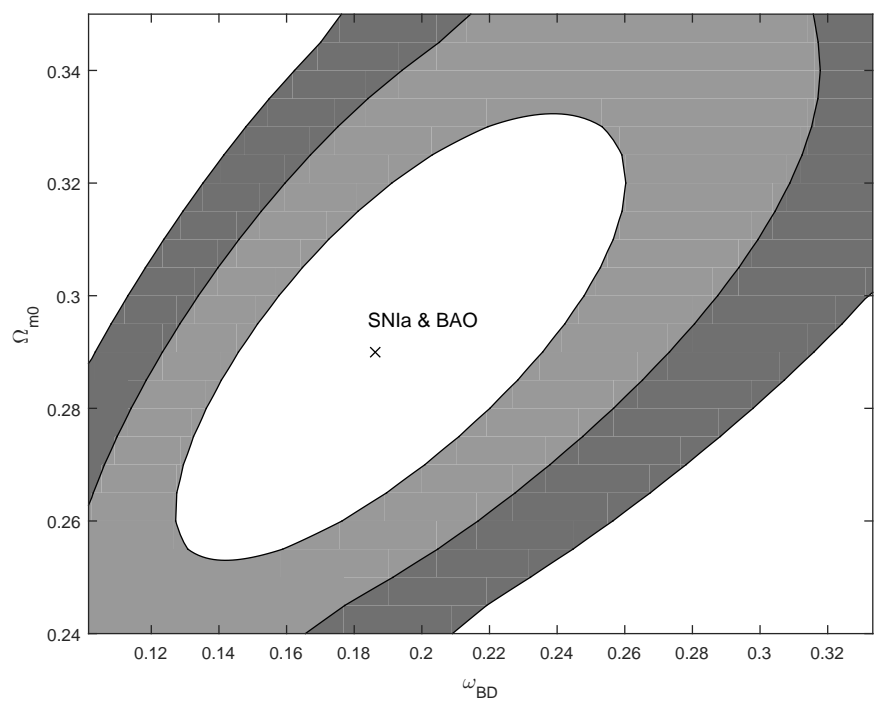

FIG. 2: The combined (SNIa+BAOs) likelihood contours $1 \sigma\left(\Delta \chi^{2}=2.3\right), 2 \sigma\left(\Delta \chi^{2}=6.18\right)$, and $3 \sigma\left(\Delta \chi^{2}=11.83\right)$, in the $\left(\Omega_{m 0}, \omega_{D E}\right)$ plane. In this analysis we use the Hubble parameter of Eq. (53). The cross corresponds to best fit solution.

and the Brans-Dicke parameter $\omega_{B D}$. The results hold for the case where the parameter $\omega_{B D}$ vanishes, i.e., action (1) is that of O'Hanlon theory which is equivalent with $f(R)$-gravity in the metric formalism. As a special case, a third family of power potentials is found when the perfect fluid is radiation, i.e. $w=1 / 3$.

By applying the zero-order invariants of the corresponding Lie symmetry in the WdW equation, we were able to solve the WdW equation and find the oscillatory behavior of the wave function. Furthermore, with the use of the Hamilton-Jacobi theory we reduced the Hamiltonian system, which defines the classical field equations, to a system of two first-order differential equations. That is, the field equations for that form of the potential, $V(\phi)$, form a Liouville integrable dynamical system.

As a special case for one of the integrable models, we found a closed-form solution for the Hubble function from which we saw that the Brans-Dicke field follows power-law behaviour with respect to the metric scale factor, that is, $\phi=\phi_{0} a^{M}$. In this context, the Hubble parameter is written as $H(a)=H_{0} \sqrt{\Omega_{\phi_{0}} a^{q_{1}}+\Omega_{m 0} a^{q_{2}}}$, where the parameters $q_{1,2}$ are given in terms of $\left(w, \omega_{B D}\right)$. Obviously, one may recover a similar Hubble parameter to this within the framework of general relativistic cosmology by using two different perfect fluids with constant equation of state parameters. This implies that the current Brans-Dicke gravity model is cosmologically equivalent to that of general relativity as far as the cosmic expansion is concerned. Therefore, in order to distinguish Brans-Dicke gravity from GR we need to extend the analysis to the perturbation level. It is well known that modified gravity affects, via the effective gravitational constant the growth rate of linear matter perturbations. As an example, in the case of $f(R)$ gravity models the quantity $G_{e f f}$ is given in terms of the scale factor and of the wave-number (see [64, 67] and references therein). In a forthcoming paper, we attempt to investigate the impact of the Brans-Dicke gravitational parameter $G_{e f f}=\left[\left(3(1+M)-\frac{\omega_{B D} M}{2}\right) \phi\right]^{-1}$ on the matter perturbations. Lastly, performing a joint statistical analysis involving the recent SNIa and BAO data, we place constraints on the main cosmological parameters of the Brans-Dicke model.

It has also been shown that there exists a relation between the value of the conserved quantity, $I_{0}$, for the classical field equations and the "frequency", $\beta$, in the WdW equation. Hartle proposed that strong peaks in the wavefunction lead to a classical observable universe [66]. Since the WdW equation is a linear second-order PDE, the general invariant solution is the sum on all possible values of $\beta$. However, one may relate the "frequency" of the strong peaks of the wavefunction to the value of the conserved quantity, $I_{0}$, (41). The latter is included in the solution of the field equations, i.e. $a(t)$, and consequently in the Hubble function $H(a)$. In any case, the existence of the conservation law indicates a strong relation between the classical and the quantum solutions and information can be transferred from among the two systems. However, the physical observable quantities which correspond to the conservation laws of the field equations are still unknown.

The Brans-Dicke action is defined in the Jordan frame and is conformally equivalent to the minimally coupled scalar field in the Einstein frame. The solutions which we have found describe a perfect fluid which is not interacting with the scalar field in the Jordan frame. However, the solutions can be transformed into the Einstein frame and will hold for a model in which there exists an interaction between the perfect fluid and the scalar field of a particular form. By definition, the two models share the same solution of the WdW equation. 
In a forthcoming work we will study the cosmological evolution of these integrable models in the Einstein frame.

\section{Acknowledgments}

AP thanks the University of Athens for the hospitality provided there when this work done. AP is supported by FONDECYT postdoctoral grant no. 3160121. SB acknowledges support by the Research Center for Astronomy of the Academy of Athens in the context of the program "Tracing the Cosmic Acceleration". JDB is supported by the STFC.

11] B. Ratra and P.J.E. Peebles, Phys. Rev. D 37, 3406 (1988)

[2] J.D. Barrow and P. Saich, Class. Quant. Grav. 10, 279 (1993)

[3] E.V. Linder, Phys. Rev. Lett. 90, 091301 (2003)

[4] E.J. Copeland, M. Sami and S. Tsujikawa, Int. J. Mod. Phys. D 15, 1753 (2006)

[5] J.M. Overduin and F.I. Cooperstock, Phys. Rev. D 58, 043506 (1998)

[6] S. Basilakos, M. Plionis and J. Sola, Phys. Rev. D 80, 083511 (2009)

[7] L. Amendola, M. Baldi and C. Wetterich, Phys. Rev. D 78, 023015 (2008)

[8] Y.F. Cai, E.N. Saridakis, M.R. Setare and J.Q. Xia, Phys. Rept. 493, 1 (2010)

[9] M.C. Bento, O. Bertolami and A.A. Sen, Phys. Rev. D 66, 043507 (2002)

[10] C. Brans and R.H. Dicke, Phys. Rev. 124, 195 (1961)

[11] H.A. Buchdahl, Mon. Not. Roy. Astron. Soc. 150, 1 (1970)

[12] T.P. Sotiriou and V. Faraoni Rev. Mod. Phys. 82, 451 (2010)

[13] R. Ferraro and F. Fiorini, Phys. Rev. D 75, 084031 (2007)

[14] R. Maartens, Living Rev. Rel. 7, 7 (2004)

[15] V. Faraoni, Cosmology in Scalar-Tensor Gravity, Fundamental Theories of Physics vol. 139, (Kluwer Academic Press: Netherlands, 2004)

[16] L. Amendola and S. Tsujikawa, Dark Energy Theory and Observations, (Cambridge University Press: Cambridge, 2010)

[17] J.D. Barrow, Phys. Lett. B 235, 40 (1990)

[18] A.G. Muslinov, Class. Quantum Gravit. 13, 3229 (1996)

[19] G.F.R. Ellis and M.S. Madsen, Class. Quantum Gravit. 8, 667 (1991)

[20] J.G. Russo, Phys. Lett. B 600, 185 (2004)

[21] S. Basilakos and G. Lukes-Gerakopoulos, Phys. Rev. D 78, 083509 (2008)

[22] J.J. Halliwell, Phys. Lett. B 185, 341 (1987)

[23] R. Easter, Class. Quantum Gravit. 102203 (1993)

[24] L.P. Chimento and A.S. Jakubi, Int. J. Mod. Phys. D 5, 71 (1996)

[25] C. Rubano and J. D. Barrow, Phys. Rev. D. 64, 127301 (2001)

[26] L. A. Urena-Lopez, T. Matos, Phys. Rev. D 62, 081302 (2000)

[27] V. Sahni and A. Starobinsky, Int. J. Mod. Phys. D 9, 373 (2000)

[28] S. Basilakos, M. Tsamparlis and A. Paliathanasis, Phys. Rev. D 83, 103512 (2011)

[29] A. Paliathanasis, M. Tsamparlis and S. Basilakos, Phys. Rev. D 90, 103524 (2014)

[30] S. Capozziello, R. de Ritis and A. A. Marino, Class. Quantum Gravity, 14, 3259 (1997); C. Rubano, and P. Scudellaro, Gen. Relat. Grav. 34, 307, (2002); A.K. Sanyal, B.Modak, C. Rubano and E. Piedipalumbo, Gen. Relat. Grav. 37, 407, (2005); M. Szydlowski et al., Gen. Rel. Grav., 38, 795, (2006); S. Capozziello, A. Stabile, and A. Troisi, Class. Quant. Grav., 24, 2153, (2007); A. Bonanno, G. Esposito, C. Rubano and P. Scudellaro, Gen. Rel. Grav., 39, 189, (2007); S. Capozziello, E. Piedipalumbo, C. Rubano, and, P. Scudellaro, Phys. Rev. D., 80, 104030, (2009); B. Vakili, Phys. Lett. B., 664, 16, (2008); Yi Zhang, Yun-gui Gong and Zong-Hong Zhu, Phys. Lett. B., 688, 13, (2010); Hao Wei, Xiao-Jiao Guo and Long-Fei Wang, Phys. Lett. B., 707, 298, (2010)

[31] A. Paliathanasis, M. Tsamparlis and S. Basilakos, Phys. Rev. D 84, 123514, (2011).

[32] S. Basilakos, S. Capozziello, M. De Laurentis, A. Paliathanasis, M. Tsamparlis, Phys. Rev. D., 88, 103526 (2013)

[33] A. Paliathanasis, M. Tsamparlis, S. Basilakos and S. Capozziello, Phys. Rev. D 89, 063532 (2014)

[34] A. Paliathanasis, M. Tsamparlis, S. Basilakos and J.D. Barrow, Phys. Rev. D 91, 123535 (2015)

[35] A. Yu Kamenshchik, E.O. Pozdeeva, A. Tronconi, G. Venturi and S. Yu Vernov, Class. Quantum Gravit. 31, 105003 (2014)

[36] E. Ahmadi-Azar and N. Riazi, Astr. Sp. Sci. 226, 1 (1995)

[37] R.E. Morganstern, Phys. Rev. D 4, 946 (1971)

[38] R.N. Tiwari and B.K. Nayak, J. Phys. A: Math. Gen. 9, 369 (1976)

[39] H. Kim, Phys. Rev. D 60, 024001 (1999)

[40] N. Riazi and H.R. Askari, Mon. Not. R. Astron. Soc. 261, 229 (1993)

[41] T. Clifton, J.D. Barrow and R.J. Scherrer Phys. Rev. D 71, 123526 (2005)

[42] Y. Kucukakca, U. Camci and I. Semiz, Gen. Rel. Gravit., 44, 1893 (2012) 
[43] P.A. Terzis, N. Dimakis and T. Christodoulakis, Phys. Rev. D. 90, 123543 (2014)

[44] S. Kamilya and B. Modak, Gen. Rel. Gravit. 36, 674 (2004)

[45] S. Capozziello, S. Nesseris and L. Perivolaropoulos, JCAP 2012 12(9) (2012)

[46] T. Clifton and J.D. Barrow, Phys. Rev. D 73, 104022 (2006)

[47] T.P. Sotiriou, Gravity and Scalar fields, Proceedings of the 7th Aegean Summer School: Beyond Einstein's theory of gravity, Modifications of Einstein's Theory of Gravity at Large Distances, Paros, Greece, ed. by E. Papantonopoulos, Lect.Notes Phys. 892 (2015)

[48] B.S. DeWitt, Phys. Rev. 1601113 (1967)

[49] J.B. Hartle and S.W. Hawking, Phys. Rev. D 282960 (1983)

[50] D. Wiltshire, An introduction to quantum cosmology (2001) (arXiv: gr-qc/0101003);

[51] J.J. Halliwell, Introductory lectures on quantum cosmology (2009) (arXiv: 0909.2566 [gr-qc])

[52] P.J. Oliver, Applications of Lie Groups to Differential Equations, Graduate Texts in Mathematics, Volume 107, (SpringerVerlag: New York, 1993)

[53] N.H. Ibragimov, Transformation Groups Applied to Mathematical Physics Mathematics and Its Applications, Soviet Series, (D Reidel Publishers: Dordrecht, 1985)

[54] E. Noether, Invariante Variationsprobleme, Nachr. v.d. Ges. d. Wiss. zu Göttingen, 235 (1918)

[55] A. Paliathanasis and M. Tsamparlis, Int. J. Geom. Meth. Mod. Phys. 111450037 (2014)

[56] J. O'Hanlon, Phys. Rev. Lett. 29137 (1972)

[57] J.D. Barrow and S. Cotsakis, Phys. Lett. B 214, 515 (1988)

[58] A. Paliathanasis, J. Phys.: Conf. Ser. 453012009 (2013)

[59] J.D. Barrow and A.C. Ottewill, J. Phys. A 16, 2757 (1983)

[60] N. Suzuki et.al, Astrophys. J. 74685 (2012)

61] W.J. Percival et al, Mon. Not. R. Astron. Soc., 4012148 (2010)

[62] C. Blake et al., Mon. Not. R. Astron. Soc. 4181707 (2011)

[63] C.L. Bennet, D. Larson, J.L. Weiland and G. Hinshaw, Ap. J. 794135 (2014)

[64] S. Basilakos, S. Nesseris and L. Perivolaropoulos, Phys. Rev. D. 87123529 (2013)

[65] H. Akaike, IEEE Transactions of Automatic Control, 19, 716 (1974); N. Sugiura, Communications in Statistics A, Theory and Methods, 7, 13 (1978)

[66] J.B. Hartle, Gravitation in Astrophysics: Cargèse 1986, Proceedings of a Nato Advanced Study Institute on Gravitation in Astrophysics, Cargèse, France, Ed. by B. Carter and J.B. Hartle, (Plenum: New York, 1986)

[67] R. Gannouji, B. Moraes and D. Polarski, JCAP, 62, 034 (2009); S. Tsujikawa, R. Gannouji, B. Moraes and D. Polarski, Phys. Rev. D., 80, 084044 (2009) 